\title{
A method to construct the dynamic landscape of a bio-membrane with experiment and simulation
}

\section{Albert Smith ( $\nabla$ albert.smith-penzel@medizin.uni-leipzig.de )}

Leipzig University https://orcid.org/0000-0002-9372-7297

\section{Alexander Vogel}

Leipzig University

\section{Oskar Engberg}

Leipzig University

\section{Peter Hildebrand}

Institute of Medical Physics and Biophysics, University Leipzig https://orcid.org/0000-0003-0063-1104

\section{Daniel Huster}

Leipzig University https://orcid.org/0000-0002-3273-0943

\section{Article}

Keywords: Biomolecular Function, NMR Relaxation, Molecular Dynamics Simulation Data, Dynamics Detector Method, Timescale-sensitive Techniques

Posted Date: July 7th, 2021

DOl: https://doi.org/10.21203/rs.3.rs-645823/v1

License: (c) (i) This work is licensed under a Creative Commons Attribution 4.0 International License. Read Full License

Version of Record: A version of this preprint was published at Nature Communications on January 10th, 2022. See the published version at https://doi.org/10.1038/s41467-021-27417-y. 


\title{
A method to construct the dynamic landscape of a bio-membrane with experiment and simulation
}

\author{
Albert A. Smith ${ }^{1 *}$, Alexander Vogel ${ }^{1}$, Oskar Engberg ${ }^{1}$, Peter Hildebrand $^{1}$, Daniel Huster ${ }^{1}$ \\ ${ }^{1}$ Institute for Medical Physics and Biophysics, Leipzig University, Härtelstr. 16-18, 04107 \\ Leipzig, Germany
}

*E-mail: albert.smith-penzel@medizin.uni-leipzig.de

\begin{abstract}
Biomolecular function is based on a complex hierarchy of molecular motions. While biophysical methods can reveal details of specific motions, a concept for the comprehensive description of molecular dynamics over a wide range of correlation times has been unattainable. Here, we report a novel approach to construct the dynamic landscape of biomolecules, which describes the aggregate influence of multiple motions acting on various timescales and on multiple positions in the molecule. To this end, we use NMR relaxation and molecular dynamics simulation data for the characterization of lipid membranes, the most important biological interface. We develop a dynamics detector method that yields site-specific amplitudes, separated both by type and timescale of motion. This separation allows the detailed description of the dynamic landscape, which reveals vast differences in motion depending on molecular position. More generally, the method is applicable to a broad range of molecular systems, and can be adapted to other timescalesensitive techniques.
\end{abstract}


Biomolecular function is determined, ultimately, by dynamics. Structure provides significant clues as to what a biomolecule might do, but ideally we want to see the molecule actually do it. Henzler-Wildman and Kern say that the "dream is to 'watch' proteins in action,"1 and point out that molecular dynamics (MD) simulation is unique in its ability to provide the time-resolved motion of atoms. Still, simulations are limited in sampling the conformational space and by inaccuracies in simulation parameters and thus require experimental validation; even then, it is not trivial to connect measured parameters to motion in a simulation. Considerable complexity arises due to different modes of motion: local librations, rotations around bonds, reorientations of molecular domains and the entire molecule, and collective motions of molecular assemblies contribute to dynamics. ${ }^{2}$ Understanding biological systems requires determining which of these motions contribute to function, remembering that this contribution can be direct or indirect. Thus, to understand function it is necessary to comprehensively describe dynamics of a molecular system, requiring separation and parameterization of multiple contributions using all experimental and MD data available.

Nuclear magnetic resonance (NMR) is powerful because experiments provide sitespecific motional information, where bond reorientation modulates interaction tensors (e.g. dipolar/quadrupolar couplings). 3,4 Measurement of one-bond residual couplings provides an order parameter, $|S|$, defined as $\left|\delta_{\text {resid. }} / \delta_{\text {rigid }}\right|$ where $\delta_{\text {resid. }}$ is the averaged anisotropy of a coupling divided by the rigid limit of the coupling. Then, all motions in a molecule leading to reorientation of the interaction contributes to the reduction of $|S|$ from 1 , albeit with a somewhat complex dependence on orientations sampled.

$|S|$ does not provide timescale resolution, whereas NMR relaxation rate constants are proportional to $1-S^{2}$ and are selective for motions having correlation times $\left(\tau_{c}\right)$ matched to the eigenfrequencies $(\omega)$ of the spin-system $\left(\omega \tau_{c} \approx 1\right)$; these frequencies can be varied by choice of experiment. This timescale selectivity helps in separating motions, but for complex systems, a complete parameterization is rarely possible, and parameterization using simplified models often creates bias. ${ }^{5}$ Alternatively, using an experimentally validated MD simulation, one should be able to extract and parameterize the specific motions. To attempt this, we consider a critical biological system: the lipid membrane, for which the complex dynamics of an extended molecular system is encoded into the reorientational motions of the single lipids.

The lipid membrane is nature's most important interface. It maintains a barrier function and provides the environment for various biological functions, i.e. communication 
and transport often mediated by embedded proteins. ${ }^{6-8}$ To enable these functions, lipid molecules are characterized by a highly dynamic structural polymorphism resulting in a well-balanced equilibrium of order and disorder. ${ }^{9}$ This is best described by a dynamic landscape in which the crucial parameters are the correlation times of motion, their distribution widths, and the motional amplitude, where multiple motions yield a product of distributions. While spectroscopic tools and MD simulations have described individual aspects of this versatile dynamics, ${ }^{10-14}$ its comprehensive and quantitative description has not been presented. Here, we suggest an analytical method to use both NMR and MD data to quantitatively describe the dynamic landscape of a palmitoyl-oleoyl-phosphatidylcholine (POPC) bilayer. The method is based on dynamic detectors, ${ }^{5,15,16}$ which describe the timescale-specific generalized amplitude of motion of the $\mathrm{C}-\mathrm{H}$ bonds of the POPC molecule.

In order to characterize the full dynamic landscape, we perform an extensive comparison of NMR/MD data using detector analysis, a method developed to eliminate bias while providing quantitative, timescale-specific comparison of motions between different methods. ${ }^{5,15}$ Next, we develop a novel frame analysis, which allows separating multiple types of motion using MD, and explicitly fit distributions of correlation times for each motion, using only a few parameters. Combining the motions, we obtain a detailed characterization of the multidimensional dynamic landscape of a lipid membrane over several decades of correlation times. This methodology is well suited to quantitatively describe membrane dynamics in response to membrane protein function, lipid domain structure, and binding of molecules to the bilayer, and may be more generally extended to the characterization of other molecular systems.

\section{Results}

A series of 7 NMR relaxation experiments $\left({ }^{13} \mathrm{C} T_{1}\right.$ at 3 fields, heteronuclear ${ }^{1} \mathrm{H}-{ }^{13} \mathrm{C}$ nuclear Overhauser effect, and ${ }^{13} \mathrm{C} T_{1 \rho}$ at 3 spin-lock strengths) and measurement of residual ${ }^{1} \mathrm{H}-{ }^{13} \mathrm{C}$ dipole couplings (DIPSHIFT) were performed and analyzed using the dynamics "detectors" method, ${ }^{15}$ providing bond-specific dynamics information. Detector analysis provides several detector responses, $\rho_{n}^{(\theta, S)}$, each of which characterizes motion within a specific window of correlation times $\left(\tau_{\mathbf{c}}\right)$, the window being defined by sensitivity functions, $\rho_{n}(z)\left(z=\log _{10}\left(\tau_{c} / 1 \mathrm{~s}\right)\right)$. Using a series of detectors, one sees how motion is distributed as a function of correlation time, and comparing responses of a single detector, 
one sees how motion varies across a molecule for the corresponding timescale. The detector response can be precisely defined as

$$
\begin{aligned}
& \rho_{n}^{(\theta, S)}=\left(1-S^{2}\right) \int_{-\infty}^{\infty} \theta(z) \rho_{n}(z) d z \\
& 0 \leq 1-S^{2} \leq 1, \int_{-\infty}^{\infty} \theta(z) d z=1
\end{aligned}
$$

$\left(1-S^{2}\right)$ depends on a bond's orientational sampling, usually becoming larger with total amplitude of motion, and $\theta(z)$ indicates how the total $\left(1-S^{2}\right)$ is distributed as a function of correlation time. The distribution function $\theta(z)$ may take on any form, including having only a few discrete correlation times or a complex correlation time continuum. For motion with correlation time $z=\log _{10}\left(\tau_{c} / \mathrm{s}\right)$, detector $n$ reports on this motion proportionally to $\rho_{n}(z)$.

Detector responses, $\rho_{n}^{(\theta, S)}$, are precisely defined by (1), but interpretation is more loose; they do not yield exact correlation times, and amplitude depends on correlation time, via $\rho_{n}(z)$. This initial ambiguity is deliberate: by avoiding a specific interpretation, we can quantitatively compare NMR to MD without introducing bias at the outset that could result from an incorrect model. To make this comparison, we calculate the reorientational correlation functions of $\mathrm{H}-\mathrm{C}$ bonds from an $8.4 \mu$ s simulation of a POPC bilayer of 256 lipid molecules. The correlation function similarly depends on the distribution of motion, where we assume the following functional form:

$$
C(t)=S^{2}+\left(1-S^{2}\right) \int_{-\infty}^{\infty} \theta(z) \rho_{n}(z) d z
$$

We calculate detector responses from the MD simulation using a similar approach as is applied for NMR analysis; ${ }^{17}$ details of both analyses are found in Methods. 

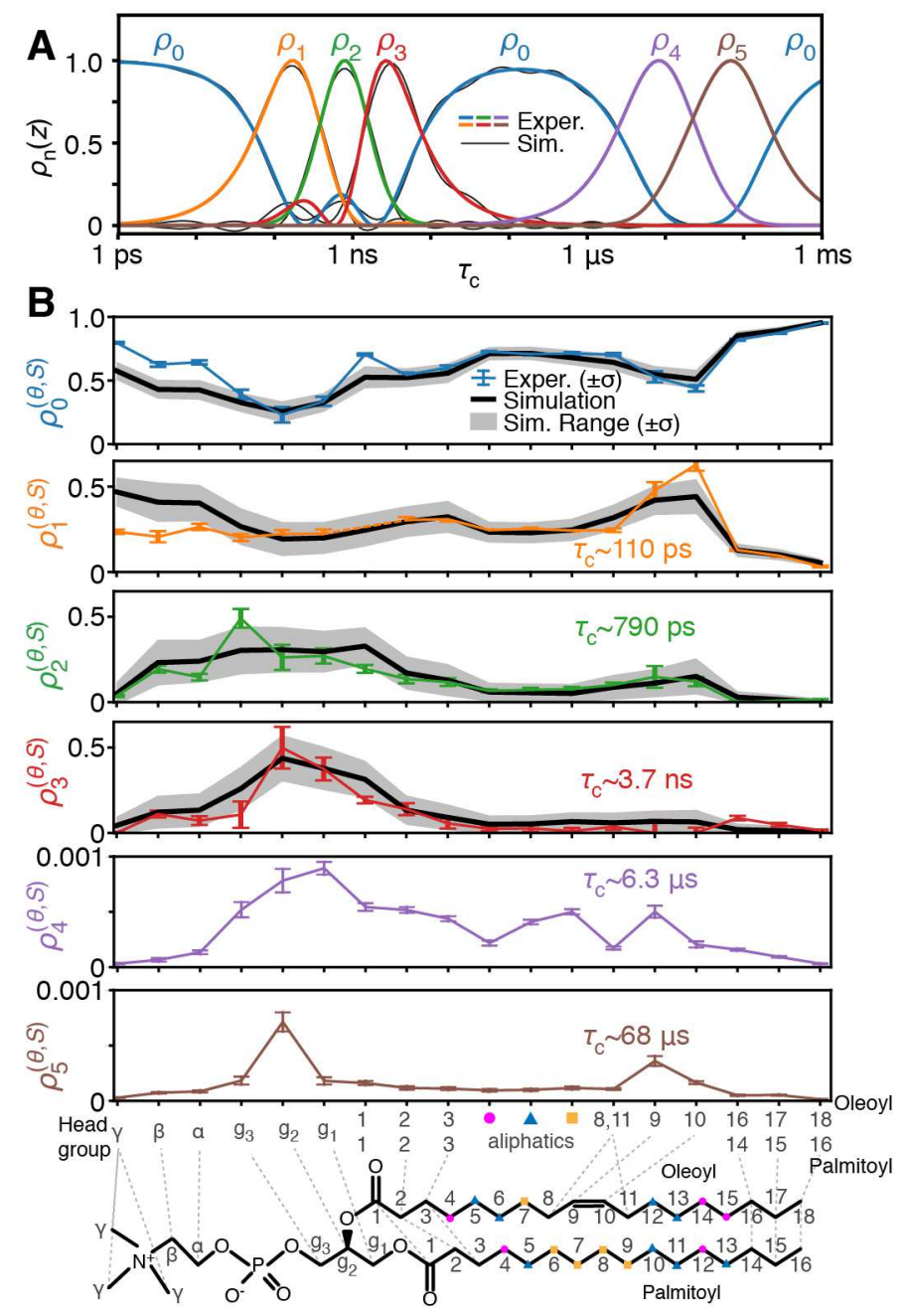

Fig. 1. Experimental vs. MD detector responses. A shows the sensitivity windows for 6 experimental detectors (color). We use MD data to approximate detectors 0-3, where the MD sensitivities are shown in grey. Detectors 4-5 are not calculated with MD. In B, the detector responses characterize the amplitude of motion in each window. Colored lines with error bars indicate the NMR detector responses and $1 \sigma$ of the experimental error, whereas black lines indicate the MD-determined amplitude and the grey range indicates $1 \sigma$ of the variation between 256 simulated molecules. Where experimental data is not resolved over multiple carbons, we average together the simulated data for the same carbons with uniform weighting for comparison to experiment.

The results are plotted in Fig. 1, where 6 detectors are obtained for 18 signals of POPC (SI Fig. 1). Sensitivities of these detectors are shown in Fig. $1 \mathrm{~A}$, where $\rho_{1}-\rho_{3}$ cover $\mathrm{ps} / \mathrm{ns}$ motion ( 0.1-4 ns), and $\rho_{4}-\rho_{5}$ cover $\mu \mathrm{s}(6-70 \mu \mathrm{s}) . \rho_{0}$ is sensitive to all motion falling outside the other windows. From MD, we could reproduce $\rho_{0}-\rho_{3}$, although the $8 \mu \mathrm{s}$ trajectory is too short to compare motions with correlation times in the microsecond range. Agreement between NMR and MD in Fig. $1 \mathrm{~B}$ is very good. The most significant outliers occur in the head group $(\alpha, \beta, y)$, where MD underestimates $\rho_{0}$ and overestimates $\rho_{1}$, indicating that MD underestimates the rate of motion in the head group, most likely related to force field imperfections. ${ }^{18}$ In the oleoyl and palmitoyl chains, we do not have full sitespecific resolution in NMR, but we do have excellent agreement with averaged detector responses obtained with MD (oleoyl C9/C10 are resolved in NMR). Then, we can combine 
results from $\mathrm{MD}$ in the chains (except oleoyl C9/C10) with experimental results elsewhere to illustrate molecular motion of POPC in Fig. 2.

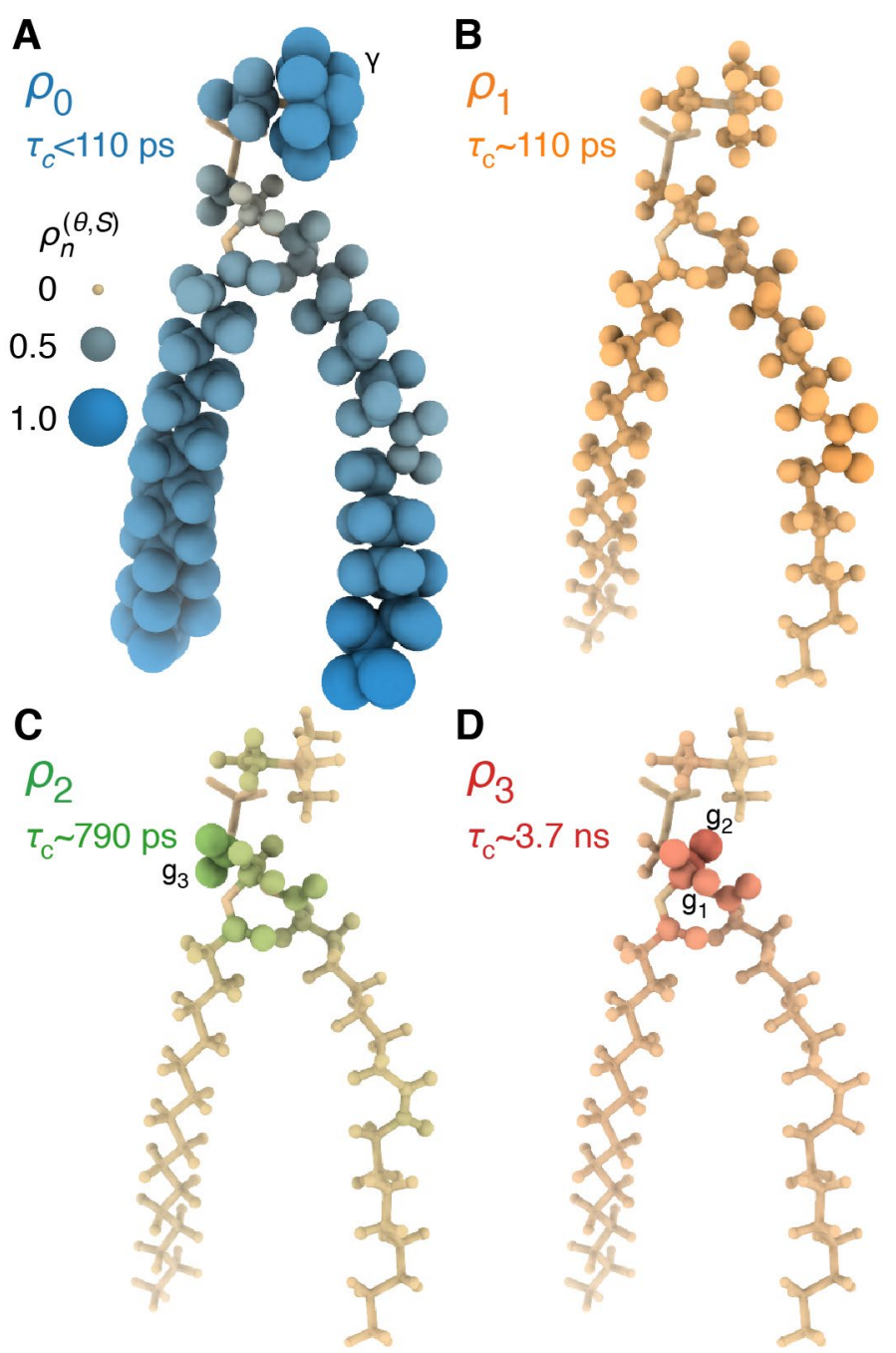

Fig. 2. Detector responses $\rho_{0}-\rho_{3}$ mapped onto the individual segments of a POPC molecule. Each plot encodes the detector responses found in Fig. 1 where the radii of the $\mathrm{H}$ and C nuclei are proportional to $\rho_{n}^{(\theta, S)}$, and we fade from tan to color, depending linearly on $\rho_{n}^{(\theta, S)}$. Radius scale is indicated in the upper left of the figure. In cases where experimental data is ambiguous (some chain methylene segments), we plot MDderived responses, noting that these responses are in very good agreement with experiment (see Fig. 1). All 3D plots produced with ChimeraX. ${ }^{19}$

In Fig. 2A, we map $\rho_{0}^{(\theta, S)}$ onto POPC. The sensitivity of $\rho_{0}$ is nonzero over multiple ranges of correlation times (Fig. 1A), but it is predominantly resulting from fast $(<110 \mathrm{ps})$ motion, which can be verified from MD analysis (SI Fig. 7). Motion is fastest at the ends of the chains and in the $\gamma$ position of the head group. From $\rho_{0}-\rho_{3}$, one sees that chain motion and head group motion is predominantly fast $\left(\rho_{0} / \rho_{1}, \sim 110\right.$ ps or faster), whereas the glycerol backbone moves significantly slower $\left(\rho_{2} / \rho_{3}\right)$. Motion is slowest for $g_{2}$ where the highest detector response occurs for $\rho_{3}(\sim 3.7 \mathrm{~ns})$. Glycerol $\mathrm{g}_{1}$ experiences similar responses for $\rho_{2}$ and $\rho_{3}$ and $g_{3}$ has the largest response for $\rho_{2}$ ( $\sim 790$ ps). To help interpret detector responses, supplementary file "det_total.mov" shows a POPC molecule from MD, 
where detector responses are plotted onto the molecule while sweeping through different timescales.

While obtaining full site resolution by combining MD and NMR is a step forward, we want to understand how different motions contribute to the overall dynamics. Therefore, we introduce a frame analysis, which allows separation of the total motion into (approximately) statistically independent components. ${ }^{20-23}$ We assume the total correlation function is given by the product of several correlation functions of independent motions:

$$
C(t)=C^{1}(t) \cdot C^{2}(t) \cdot \ldots \cdot C^{n}(t)
$$

This is achieved by defining a series of frames- a frame could be, e.g., the z-component of the moment of inertia (MOI) for a carbon chain. Then, we can calculate a correlation function for motion of a given $\mathrm{H}-\mathrm{C}$ bond within the frame by rotating each frame of the trajectory such that the longest (z-) component of the MOI always lies on the z-axis, and evaluate the resulting $\mathrm{H}-\mathrm{C}$ motion. Subsequently, we may determine how motion of the frame results in the $\mathrm{H}-\mathrm{C}$ bond motion, and calculate the corresponding function. The product of the resulting correlation functions is the total correlation function if: 1) terms resulting from motion within the frame are statistically independent from terms resulting from motion of the frame, and 2) significant reorientation/reshaping of the residual NMR tensor brought about by motion within the frame occurs on a timescale significantly faster than motion of the frame. Changes in tensor magnitude only are not subject to timescale separation. This procedure may be implemented iteratively to separate multiple correlation functions. It is an extension of the Lipari-Szabo model-free approach ${ }^{21,24}$ and related to the two-step approach of Wennerström and co-workers. ${ }^{22,25,26}$ Extension of model-free theory from a theoretical principle to explicit implementation is detailed in Methods.

Using frame analysis, for each bond, we separate 3-4 independent motions (Fig. $3 \mathrm{~A})$. To separate one-bond librations, we define a frame that aligns the $C$ of the bond and all directly bonded atoms to a reference structure in order to capture local structural distortions. For the glycerol backbone ( $g_{1}, g_{2}$, and $\left.g_{3}\right)$, head group $(\alpha, \beta, y)$, and carbonyls (together abbreviated $\mathrm{HG} / \mathrm{BB}$ ), we define a frame that aligns the glycerol carbons and oxygens to separate overall motion from internal structural changes of the HG/BB. Similarly, in the chains (excluding carbonyls), we define a frame that aligns the longest (z-) components of the $\mathrm{MOI}$ (approximately the direction that chain points) to separate internal reorientation from overall motion of the chain. Within the chains (excepting oleoyl carbons 9/10), we furthermore separate motion into components parallel and perpendicular to the MOI. 

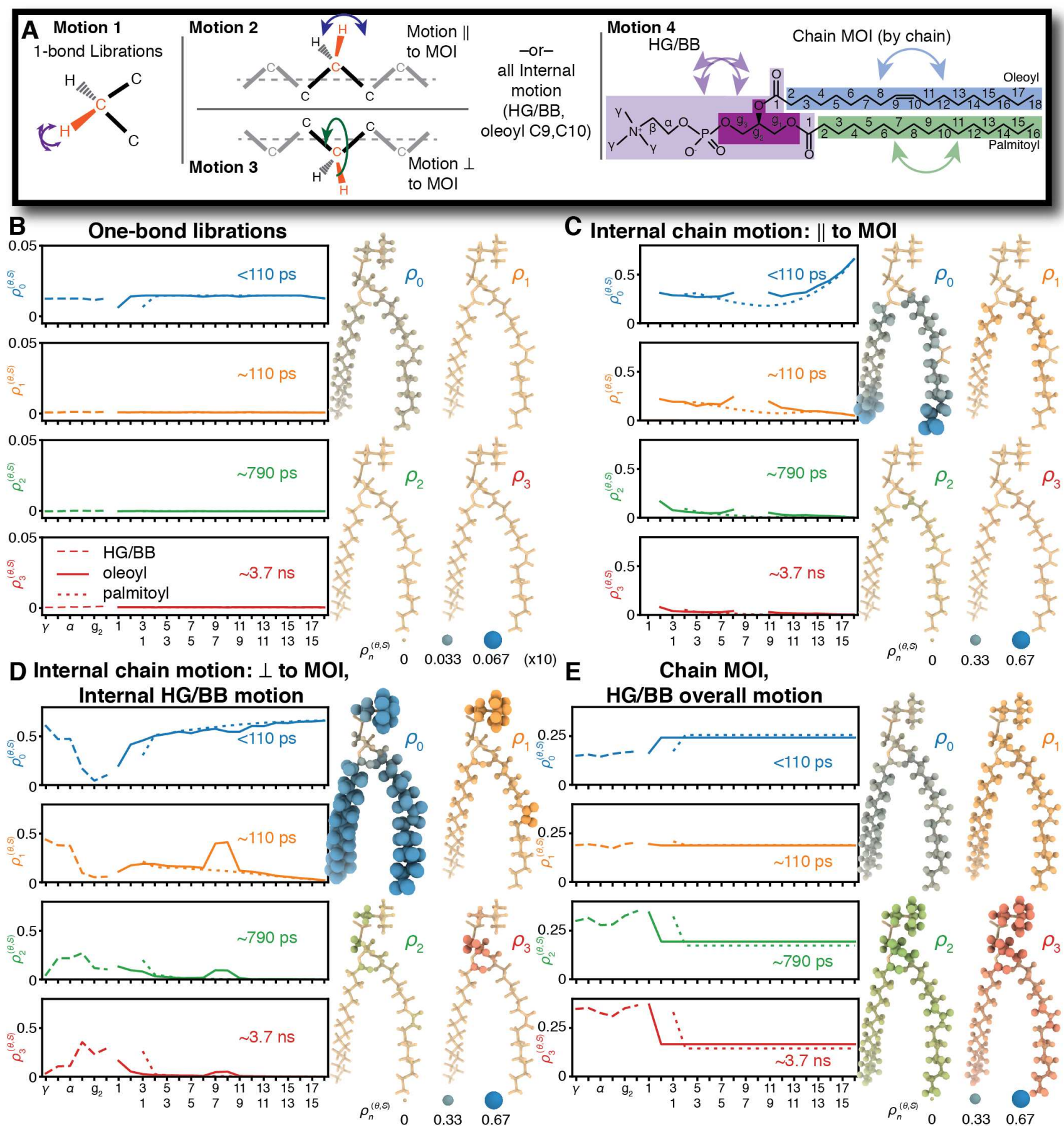

Fig. 3. Detector analysis of individual frames. A illustrates the types of motion captured by the frame analysis: One-bond $\mathrm{H}-\mathrm{C}$ (or $\mathrm{C}=\mathrm{O}$ ) librations are separated for all sites. Within chains, we separate motion parallel to the moment of inertia (MOI) and perpendicular to the MOI. For the head group, C', and oleoyl carbons 9,10 (double-bonded), we do not separate internal motion into two components. Overall motion of HG/BB is defined by the alignment of the glycerol atoms to a reference structure, and overall motion of the chains is defined by motion of the longest component (z-component) of each chain's MOI. B-E plot the detector analysis of the motions of each of the four motions, with detector responses also encoded onto the POPC molecule, where color intensity and radius depending on the detector response (same scale for C-E, scale $\mathrm{x} 10$ in B). For motion not split into perpendicular/parallel components, the internal motion (without libration) is shown in $\mathbf{D}$, with no data shown in $\mathbf{C}$.

Results of the frame analysis (Fig. 3B-E) show varying behavior for the different motions. Responses from one-bond librations (Fig. 3B) are significantly smaller than all other motions, and found exclusively in $\rho_{0}\left(\tau_{c}<110 \mathrm{ps}\right)$. Internal motions (Fig. 3C/D) are slower, with the largest responses for $\rho_{0} / \rho_{1}$, with the exception of the glycerol backbone, 
where motion is predominantly found in $\rho_{2} / \rho_{3}(790 \mathrm{ps} / 3.7 \mathrm{~ns})$, being about $10 x$ slower than other internal motion. Within the chains, motion perpendicular to the chain's $\mathrm{MOI}$ is slightly faster and typically higher amplitude than motion parallel to the MOI. Overall motion (HG/BB or chain motion) differs considerably from internal motion: on average it is more broadly distributed (all $\rho_{n}^{(\theta, S)}$ have similar amplitudes), with significantly slower components, since $\rho_{3}^{(\theta, S)}(\sim 3.7 \mathrm{~ns})$ becomes large. Individual motions and detector responses can be viewed as movies in supplementary files "det_*.mov".

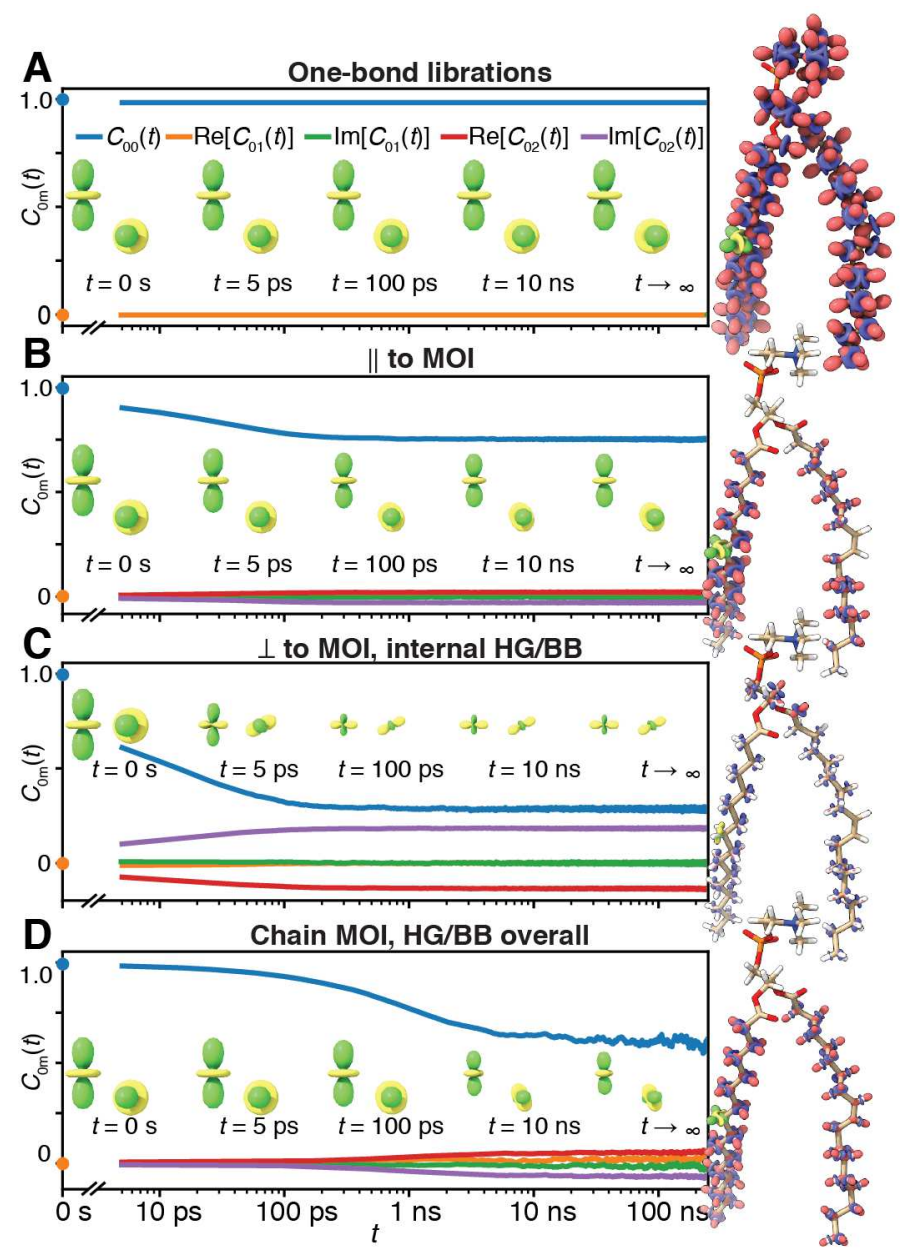

Fig. 4. Tensor reorientation due to motion. A-D plot the residual tensors onto the POPC molecule resulting from individual motions A: librational motion. B: motion parallel to the moment of inertia (no data for $\mathrm{HG} / \mathrm{BB}$ and oleoyl carbons 9,10). C: motion perpendicular to the MOI (chains), or all internal motion (one-bond librations removed) for $\mathrm{HG} / \mathrm{BB}$, and oleoyl carbons 9,10. D: overall motions (chains defined by z-axis of $\mathrm{MOI}$, HG/BB by RMS alignment of glycerol group). In A-D, pictures within the plots show the time dependence of the palmitoyl C8 tensor reorientation (green: positive, yellow: negative); the plots themselves show correlation functions defining the orientation of the C8 tensor $\left(C_{0 p}(t)=\left\langle D_{0 p}^{2}\left(\Omega_{\tau \neq+\tau}^{\text {vif-F }}\right)\right\rangle_{\tau}\right.$, see Methods). In the molecule plots (right), red indicates the positive part of the tensor, and blue the negative part. C8 (palmitoyl) is highlighted in the molecule plots in green and yellow.

Detector analysis of individual motions captures contributions to $1-S^{2}$ as a function of timescale. However, each motion samples bond orientations differently, which is not easily seen in Fig. 3. In NMR, anisotropic interactions such as dipolar tensors are averaged by orientational sampling, resulting in a residual tensor of the interaction. This may be 
probed directly via measurement of residual couplings to study the total molecular motion. However, it is not simple to separate individual motions' contributions to the residual tensor, and the sign and spatial orientation of the residual tensor cannot be accessed with powderaveraged samples. Furthermore, the residual tensor from faster motion affects the relaxation induced by slower motion (see Methods for details). Therefore, in Fig. 4, we illustrate residual tensors resulting from each motion (estimated shape as $t \rightarrow \infty$ ), including time dependence for one carbon. Evolution of all tensors is shown in the supplementary files "tensor_*.mov". Since librational motion has very low amplitude, it results in minimal change to the residual tensor shape. Motion parallel to the chain $\mathrm{MOI}$ results in decreasing the longest component of the tensor, and introduces anisotropy (Fig. 4B). This is expected, since the parallel motion is a restricted rotation around one axis; sampling of a wider range of angles would result in a smaller tensor with larger anisotropy. Motion perpendicular to the $\mathrm{MOI}$ is a symmetric rotation around the $\mathrm{MOI}$, resulting in significant reorientation of each tensor such that all residual tensors in chains align parallel to the $\mathrm{MOI}$ (Fig. 4C). Overall motion of the HG/BB causes residual tensors to nearly vanish, whereas MOI motion of the chains reduces tensor magnitudes by about 50\% (Fig. 4D). Clearly, the frame analysis provides unprecedented insights into the molecular details of the motion of POPC in membranes and how motion relates to experimentally measurable parameters.

In Fig. 1-2 we obtain a characterization of the distribution of motion, $\left(1-S^{2}\right) \theta(z)$. However, attempting to estimate the specific form of $\left(1-S^{2}\right) \theta(z)$ is unlikely to return good results, due to the presence of multiple motions, each having different characteristics and therefore requiring many parameters to describe. Once we separate the total motion into components, it is more reasonable to assume a simple functional form for the distribution of motion of each component. Then, detector responses for each bond, and each motion are fitted to a 3-parameter model of the distribution of motion, where parameters are correlation time, order parameter $\left(1-S^{2}\right)$, and width. A skewed distribution is used for internal motion, as might be expected for power-law behavior (collective motions ${ }^{27,28}$ ), and a regular Gaussian distribution for overall (HG/BB and chain MOI) motions.

The resulting MD-derived distributions may be found in SI Fig. 14, and we plot the total distribution of motion in SI Fig. 16, which results from the product of correlation functions (eq. (3)). Although MD agrees well with experiment (Fig. 1), we would like to perform a final refinement based on experimental results. To achieve this, we adjust the internal correlation time for each resonance. Where multiple positions in the POPC molecule overlap in the spectrum, we scale each correlation time by the same factor. Similarly, in the chains, we scale parallel and perpendicular components equally. 
Parameters from MD only and including experimental refinement are in SI Fig. 13, and improvement in experimental agreement due to refinement is shown in SI Fig. 17.

Experimentally refined distributions of motion are shown in Fig. 5A-D. While one cannot easily extract distributions from detectors describing overall motion, at the risk of losing important details of the individual motions, one may determine the total distribution by combining contributions from the individual motions; the result should satisfy the product in eq. (3) (see Methods). The resulting dynamic landscape of POPC is shown in Fig. 5E.

A

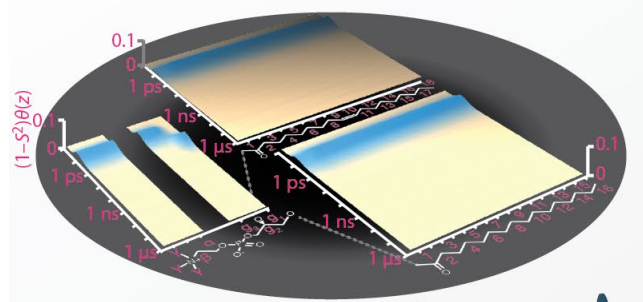

One-bond librations

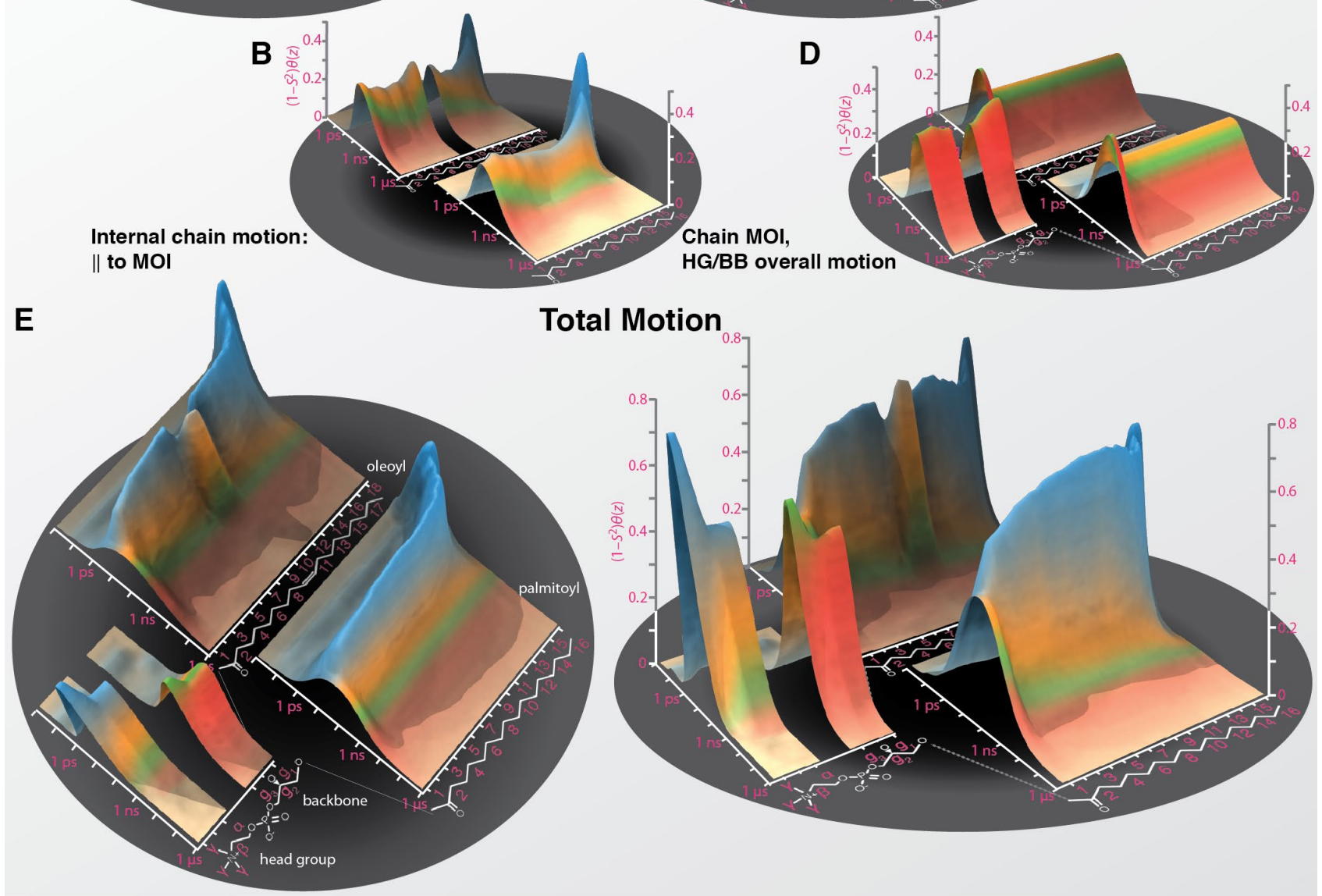

C

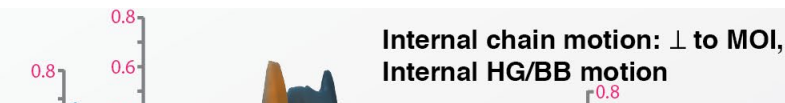

Fig. 5. Dynamic landscape of POPC membranes. A-D plot fitted distributions of motion for the separated motion. Axes are the correlation time (left), and position (right), where each plot is broken up into parts (left: HG/BB, back: oleoyl, front: palmitoyl). The coloring corresponds to the experimental detector most sensitive to a given correlation time (blue: $\rho_{0}$, orange: $\rho_{1}$, green: $\rho_{2}$, red: $\rho_{3}$ ), where the intensity of the color is determined by the amplitude at the corresponding correlation time (fades to tan for small amplitudes). $\mathbf{E}$ plots the distribution of motion for the total motion, resulting from the product of correlation functions corresponding to the distributions in A-D (eq. (3)). Details of fitting and calculating the total distribution given in SI section 4 . 


\section{Discussion}

Detector analysis provides a general method to quantitatively describe the dynamic landscape on which biomolecules exist based on experimental and/or simulated data. Detectors describe the timescale-specific generalized amplitude of motion. If a sufficient number of detectors can be extracted from experimental and MD data, while separating motions with frames, the full dynamic landscape of a biomolecule can be constructed. Doing so, we rely on the detail provided by MD simulation, but refine results with the better accuracy of experiments.

The dynamics landscape (Fig. 5) brings the broad range of motion in the POPC membrane into stark relief, while still connecting that motion to the experimentally derived detector responses. The total amplitude of motion, $1-S^{2}$, which can be obtained by integrating over correlation time, relates the molecular flexibility (the range of orientations sampled), whereas correlation time $\left(\tau_{c}\right)$ reports the mobility (how quickly those orientations are sampled), both obtained as a function of molecular position. ${ }^{28}$ In terms of the energetic landscape, amplitude informs us about the thermal equilibrium between states: a large value for $\left(1-S^{2}\right)$ implies that bonds undergo significant sampling of a large range of orientations, and these orientations must have similar free energy. Shorter correlation times indicate that the free energy cost of transitions between those orientations is low.

Consider the differences that can be seen in Fig. 5E: at the end of the chains and the head group, we have both high amplitude and short correlation times, so that the almost all orientations are sampled, and the energetic cost of doing so is low (SI Fig. 14). Contrast this to the backbone: amplitudes are slightly lower (similar to the middle of the palmitoyl chain, see $1-S^{2}$ in SI Fig. 13), but correlation times are much longer. Internal backbone motion (Fig. 5C) has a much higher free energy cost than internal chain motion. In SI Fig. 18 , we see that this cost comes from hops between several configurations of the backbone. This, however, is only a fraction of the total motion, the remainder coming from overall HG/BB motion (Fig. 5D), which is similarly slow. In this case, the energetic cost comes from collectivity of motion: ${ }^{29}$ concerted motions of many molecules in the membrane. This free energy is the sum of high enthalpy due to the large number of molecules involved and the entropic cost of the motion happening in a concerted fashion. Aside from long $\tau_{c}$, the collective motion also causes a broad distribution over correlation time. Collective motions do not happen over a fixed distance-we may have both short- and long-distance modes of motion-therefore we also observe a broad distribution of correlation times, with longer correlation times corresponding to longer distance motion. This leads to an essential 
property of the membrane: its elasticity. ${ }^{30,31}$ While the backbone undergoes significant motion, a large part of that motion is collective, so that it does not result in the breaking of the local membrane structure. While one finds these modes via combination of extensive field-cycling and temperature dependent experiments, ${ }^{27,32}$ even then it is not possible to cleanly separate backbone collective motion from internal motion, since they occur on the same timescale; however, separation is achieved in Fig. 5C/D.

Distributions of correlation times are also observed for internal motions: in the backbone, this results from transitions between six configurations (SI Fig. 18), and in the chains, numerous possible trans/gauche transitions also broaden the correlation time distribution. ${ }^{33,34}$ Collective dynamics also influences the chains (Fig. 5D), although from Fig. $5 \mathrm{E}$ we expect the resulting detector responses to be significantly lower. Indeed, $\rho_{2}^{(\theta, S)}$ and $\rho_{3}^{(\theta, S)}$ in Fig. 1B show progressively lower responses. Although we cannot reliably predict $\rho_{4}^{(\theta, S)}$ or $\rho_{5}^{(\theta, S)}$ with only $8.4 \mu$ s of simulation, we would expect to see that collective motions are acting on small residual tensors from faster motions. Experiment agrees: $\rho_{4}^{(\theta, S)}$ is largest in the backbone where internal motion is smallest, and so residual tensors are larger, and in fact $\rho_{5}^{(\theta, S)}$ reaches its maximum at $\mathrm{g}_{2}$ of the backbone, where internal motion is at its minimum.

Thus, on the basis of MD simulation and somewhat limited experimental data, detector analysis provides broad insight into the dynamics of POPC membranes. Access to site-specific data for multiple types of motion, their correlation times, amplitude, and breadths based on experiment alone typically would require temperature dependence and field cycling, the latter of which prevents site-specific characterization without specific isotopic labeling. ${ }^{33,35}$ Then, it is possible to validate the simulation with resolution in timescale using the detector analysis. Even so, it is not possible to fully parameterize multiple types of motion influencing $\mathrm{H}-\mathrm{C}$ bond reorientation based on $\mathrm{H}-\mathrm{C}$ correlation functions alone. Therefore, motion was decomposed using a novel frame analysis, allowing us to analyze and fit motions separately, resulting in a fully parameterized dynamic landscape in Fig. 5. The landscape, via color-coding, is still connected to the original detector windows used for experimental data analysis. This is a major strength of this method: we may always compare individual motions to total motions, and experiment to simulation using the quantitative and timescale-selective detectors.

The dynamic landscape is critical to understanding motion in a complex system, where correlation time and amplitude are intimately related to the energy landscape. Capturing the form of the dynamic landscape, however, is only possible because we are 
able to perform the initial steps of analysis with limited assumptions via detector analysis, thus avoiding biasing, and second by taking full advantage of the atom-specific information in MD to separate motion via frames, yielding simple distributions that may be reasonably parameterized. In lipid membranes, this information may be used to understand how motion of the membrane couples to and allows or restricts motion in the proteins embedded within it. We expect future studies to investigate how membrane composition, including proteins themselves, influences these motions. However, we need not focus only on the membrane; our approach is general; protein and other molecular systems may be similarly measured and characterized. We may also modify our approach, using MD to identify locations and approximate timescales of critical motions, and focus experimental efforts on those dynamics. Therefore, our approach may be the basis of a comprehensive understanding of motion and its function in complex systems.

\section{Code availability}

pyDIFRATE was used for the analyses presented here, and is available as open source software under the GNU General Public License. pyDIFRATE and additional scripts for calculations specific to this study of POPC are available on GitHub:

https://github.com/alsinmr/POPC_frames_archive.

\section{Data availability}

NMR data has been provided in the Supplementary Information. Partially processed MD data is provided as binaries included in the GitHub repository (see above). Full trajectories are available upon request, and may also be viewed via MDsrv, ${ }^{36}$ using the following links: http://proteinformatics.org/mdsrv.html?load=file://public/papers/popc_dynamics/popc_256.ngl http://proteinformatics.org/mdsrv.html?load=file://public/papers/popc_dynamics/popc_1024.ngl http://proteinformatics.org/mdsrv.html?load=file://public/papers/popc_dynamics/popc_4096.ngl

\section{Author contributions}

A.S. has developed the analysis techniques presented and performed NMR experiments. A.V. and P.H. have performed MD simulations. O.E. has prepared samples. D.H. has provided lipid expertise and helped develop the landscape concept. A.S. has prepared the final version of the manuscript with all authors providing critical feedback to the presentation and conclusions of this work. 


\section{Acknowledgements}

This study was funded by the Deutsche Forschungsgemeinschaft (DFG) through CRC 1423, project number 421152132, subproject A02, and through DFG grant SM 576/1-1.

O.E. acknowledges grants from the Sigrid Juselius Foundation and Ruth and Nils-Erik Stenbäck's Foundation

\section{References}

1. Henzler-Wildman, K. \& Kern, D. Dynamic personalities of proteins. Nature vol. 450 964972 (2007).

2. Lewandowski, J. R., Halse, M. E., Blackledge, M. \& Emsley, L. Direct observation of hierarchical protein dynamics. Science vol. 348578 (2015).

3. Palmer, 3rd, A. G. Nmr probes of molecular dynamics: overview and comparison with other techniques. Annu. Rev. Biophys. Biomol. Struct. vol. 30 129-55 (2001).

4. Schanda, P. \& Ernst, M. Studying Dynamics By Magic-Angle Spinning Solid-State NMR Spectroscopy: Principles and Applications to Biomolecules. Prog. Nucl. Mag. Res. Sp. vol. 96 1-46 (2016).

5. Smith, A. A., Ernst, M. \& Meier, B. H. Because the light is better here: correlation-time analysis by NMR spectroscopy. Angew. Chem. Int. Ed. vol. 56 13778-13783 (2017).

6. Singer, S. J. \& Nicolson, G. L. The Fluid Mosaic Model of the Structure of Cell Membranes. Science 175, 720-731 (1972).

7. Goñi, F. M. The basic structure and dynamics of cell membranes: An update of the Singer-Nicolson model. Biochim. Biophys. Acta BBA - Biomembr. 1838, 1467-1476 (2014).

8. Casares, D., Escribá, P. V. \& Rosselló, C. A. Membrane Lipid Composition: Effect on Membrane and Organelle Structure, Function and Compartmentalization and Therapeutic Avenues. Int. J. Mol. Sci. 20, 2167 (2019).

9. White, S. H., Ladokhin, A. S., Jayasinghe, S. \& Hristova, K. How membranes shape protein structure. J. Biol. Chem. 276, 32395-32398 (2001).

10. Venable, R. M., Zhang, Y., Hardy, B. J. \& Pastor, R. W. Molecular dynamics simulations of a lipid bilayer and of hexadecane: an investigation of membrane fluidity. Science $\mathbf{2 6 2}$, 223-226 (1993).

11. Huster, D., Arnold, K. \& Gawrisch, K. Investigation of Lipid Organization in Biological Membranes by Two-Dimensional Nuclear Overhauser Enhancement Spectroscopy. J. Phys. Chem. B 103, 243-251 (1999).

12. Feller, S. E., Huster, D. \& Gawrisch, K. Interpretation of NOESY Cross-Relaxation Rates from Molecular Dynamics Simulation of a Lipid Bilayer. J. Am. Chem. Soc. 121, 89638964 (1999).

13. Vermeer, L. S., de Groot, B. L., Réat, V., Milon, A. \& Czaplicki, J. Acyl chain order parameter profiles in phospholipid bilayers: computation from molecular dynamics simulations and comparison with 2H NMR experiments. Eur. Biophys. J. 36, 919-931 (2007).

14. Ferreira, T. M. et al. Cholesterol and POPC segmental order parameters in lipid membranes: solid state $1 \mathrm{H}-13 \mathrm{C}$ NMR and MD simulation studies. Phys Chem Chem Phys vol. 15 1976-89 (2013).

15. Smith, A. A., Ernst, M. \& Meier, B. H. Optimized 'detectors' for dynamics analysis in solid-state NMR. J. Chem. Phys. vol. 148045104 (2018). 
16. Smith, A. A., Ernst, M., Meier, B. H. \& Ferrage, F. Reducing bias in the analysis of solution-state NMR data with dynamics detectors. J Chem Phys vol. 151034102 (2019).

17. Smith, A. A., Ernst, M., Riniker, S. \& Meier, B. H. Localized and collective motions in HET-s(218-289) fibrils from combined NMR relaxation and MD simulation. Angew. Chem. Int. Ed. vol. 58 9483-9488 (2019).

18. Antila, H. S., M. Ferreira, T., Ollila, O. H. S. \& Miettinen, M. S. Using Open Data to Rapidly Benchmark Biomolecular Simulations: Phospholipid Conformational Dynamics. J. Chem. Inf. Model. 61, 938-949 (2021).

19. Pettersen, E. F. et al. UCSF ChimeraX: Structure visualization for researchers, educators, and developers. Protein Sci. vol. 30 70-82 (2021).

20.Brown, M. F. Membrane Structure and Dynamics Studied with NMR Spectroscopy. in Biological Membranes: A Molecular Perspective from Computation and Experiment (eds. Merz, K. M. \& Roux, B.) (Birkhäuser Basel, 1996). doi:10.1007/978-1-4684-8580-6.

21. Lipari, G. \& Szabo, A. Model-free approach to the interpretation of nuclear magnetic resonance relaxation in macromolecules. 1 . Theory and range of validity. J. Am. Chem. Soc. vol. 104 4546-4559 (1982).

22. Halle, B. \& Wennerström, H. Interpretation of magnetic resonance data from water nuclei in heterogeneous systems. J. Chem. Phys. vol. 75 1928-1943 (1981).

23. Salvi, N., Abyzov, A. \& Blackledge, M. Analytical Description of NMR Relaxation Highlights Correlated Dynamics in Intrinsically Disordered Proteins. Angew. Chem. Int. Ed. vol. 56 14020-14024 (2017).

24. Lipari, G., Szabo, A. \& Levy, R. M. Protein dynamics and NMR relaxation: comparison of simulations with experiment. Nature vol. 300 197-198 (1982).

25. Wennerstroem, H., Lindman, B., Soederman, O., Drakenberg, T. \& Rosenholm, J. B. Carbon-13 magnetic relaxation in micellar solutions. Influence of aggregate motion on T1. J. Am. Chem. Soc. vol. 101 6860-6864 (1979).

26. Halle, B. The physical basis of mode-free analysis of NMR relaxation data from proteins and complex fluids. J. Chem. Phys. vol. 131224507 (2009).

27. Nevzorov, A. A. \& Brown, M. F. Dynamics of lipid bilayers from comparative analysis of $2 \mathrm{H}$ and $13 \mathrm{C}$ nuclear magnetic resonance relaxation data as a function of frequency and temperature. J. Chem. Phys. vol. 107 10288-10310 (1997).

28. Brown, M. F. 7. Collective dynamics in lipid membranes. Characterization of Biological Membranes 231-268 (2019) doi:10.1515/9783110544657-007.

29. Nevzorov, A. A., Trouard, T. P. \& Brown, M. Correlation functions for lipid membrane dynamics obtained from NMR spectroscopy. Phys. Rev. E vol. 55 3276-3282 (1997).

30.Weisz, K., Groebner, G., Mayer, C., Stohrer, J. \& Kothe, G. Deuteron nuclear magnetic resonance study of the dynamic organization of phospholipid/cholesterol bilayer membranes: molecular properties and viscoelastic behavior. Biochemistry 31, 11001112 (1992).

31. Stohrer, J. et al. Collective lipid motions in bilayer membranes studied by transverse deuteron spin relaxation. J. Chem. Phys. vol. 95 672-678 (1991).

32. Fraenza, C. C., Meledandri, C. J., Anoardo, E. \& Brougham, D. F. The Effect of Cholesterol on Membrane Dynamics on Different Timescales in Lipid Bilayers from Fast Field-Cycling NMR Relaxometry Studies of Unilamellar Vesicles. ChemPhysChem 15, 425-435 (2014).

33. Seelig, A. \& Seelig, J. Dynamic structure of fatty acyl chains in a phospholipid bilayer measured by deuterium magnetic resonance. Biochemistry vol. 13 4839-4845 (2002).

34. Seelig, J. Deuterium magnetic resonance: theory and application to lipid membranes. $Q$. Rev. Biophys. 10, 353-418 (1977).

35. Seelig, J. \& Niederberger, W. Deuterium-labeled lipids as structural probes in liquid crystalline bilayers. Deuterium magnetic resonance study. J. Am. Chem. Soc. 96, 20692072 (1974). 
36. Tiemann, J. K. S., Guixà-González, R., Hildebrand, P. W. \& Rose, A. S. MDsrv: viewing and sharing molecular dynamics simulations on the web. Nat. Methods 14, 1123-1124 (2017). 


\section{Methods}

\section{Sample Preparation}

POPC powder (Avanti Polar Lipids, Alabaster, AL, U.S.A.) was dissolved in 1:1 Chloroform $/ \mathrm{MeOH}$, and evaporated in a rotary evaporator at $40{ }^{\circ} \mathrm{C}$. Afterward, the sample was redissolved in cyclohexane and lyophilized overnight to acquire a fluffy powder and hydrated to 50 wt\% using a HEPES buffer (10 mM HEPES, $100 \mathrm{mM} \mathrm{NaCl}, \mathrm{pH} 7.4$, prepared in Milli-Q H20). Multilamellar vesicles were produced by gentle centrifugation and 10 freeze-thaw cycles between a $40^{\circ} \mathrm{C}$ water bath and liquid nitrogen. Finally, the sample was inserted into a 3.2 or $4 \mathrm{~mm}$ NMR rotor.

\section{Simulation}

A simulation of the membrane containing 256 POPC lipids was simulated for 8.37 $\mu \mathrm{s}$. In addition, systems with 1024 and 4096 POPC lipids were simulated for $9.5 \mu$ s and 2.0 $\mu \mathrm{s}$, respectively, to verify that trajectory size does not affect the dynamics characterized in this work (SI Fig. 8). Each system was built in a rectangular periodic box and contained 42.1875 TIP3 waters ${ }^{1}$ per lipid (50 wt\%) and $0.1 \mathrm{M} \mathrm{NaCl}$. Setup of the systems was conducted using published procedures..$^{2-6}$ Each system was energy minimized with the steepest descents algorithm and $1000 \mathrm{~kJ} \mathrm{~mol}^{-1} \mathrm{~nm}^{-1}$ as the threshold. All systems were equilibrated with harmonic positional restraints applied to lipids that were sequentially released in a series of equilibration steps. For each system considerable time was spent on unbiased equilibration (500 ns) and the remaining trajectory used for analysis. Coordinates were saved every $0.5 \mathrm{ps}$. The simulations were run in the NPT ensemble at a temperature of $298.15 \mathrm{~K}$ and a pressure of 1.0 bar using GROMACS 2019.2 and newer using the CHARMM36 force field. ${ }^{7}$ Particle-mesh Ewald was used to treat electrostatic interactions, using a cut-off distance of $10 \AA$. Bonds involving hydrogen were constrained with LINCS $^{8}$ to allow a time step of $2 \mathrm{fs}$. MDsrv sessions ${ }^{9}$ of the simulations may be viewed at: http://proteinformatics.org/mdsrv.html?load=file://public/papers/popc_dynamics/popc_256.ngl http://proteinformatics.org/mdsrv.html?load=file://public/papers/popc_dynamics/popc_1024.ngl http://proteinformatics.org/mdsrv.html?load=file://public/papers/popc_dynamics/popc_4096.ngl

\section{Experimental Setup}

Eight NMR experiments were acquired to characterize the dynamic behaviour of POPC. All experiments were acquired as a series of 1D (pseudo-2D), ${ }^{13} \mathrm{C}$ detected spectra, with incrementation of a relaxation delay between 1D experiments. All experiments were 
initiated with a single pulse on ${ }^{13} \mathrm{C}$ (no polarization transfer from ${ }^{1} \mathrm{H}$ ), to avoid biasing of the measured dynamics due to dynamics effects on the polarization transfer. Pulse sequences are shown in SI Fig. 2. Critical parameters for each experiment are given in Table 1. All experiments were acquired with at least 16 dummy scans for equilibration, and SPINAL64 decoupling. ${ }^{13}$ Delays for relaxation experiments are approximately log spaced (first time point is always $0 \mathrm{~s}$, second and final time point are listed). Separate $R_{1 \rho}$ experiments with different offsets were acquired for different regions of the spectrum, to minimize variation of the effective field due to offset of the applied field. ${ }^{14}$ For experiments with $\omega_{1} / 2 \pi$ of 12.0 and $22.1 \mathrm{kHz}, 2$ separate experiments were performed, so offsets were never greater than 4.4 $\mathrm{kHz}$, and for experiments with $\omega_{1} / 2 \pi$ of $7.0 \mathrm{kHz}, 4$ separate experiments were performed, so offsets were never greater than $2.4 \mathrm{kHz}$. Field strengths for $R_{1} \rho$ experiments were verified via nutation experiments. The DIPSHIFT dephasing period used frequencyswitched Lee-Goldburg decoupling for homonuclear decoupling, ${ }^{15}$ and SPINAL-64 for heteronuclear decoupling.

Table 1: Experimental parameters

\begin{tabular}{|c|c|c|c|c|c|c|c|c|}
\hline Type & $\begin{array}{l}\text { Acq. time } \\
\text { /ms }\end{array}$ & Scans & Reps. & time pts & $\begin{array}{c}\text { 2nd/last } \\
\text { delay }\end{array}$ & $\begin{array}{c}\omega_{0} / 2 \pi\left({ }^{13} \mathrm{C}\right) \\
/ \mathrm{MHz}\end{array}$ & $\begin{array}{c}\omega_{\mathrm{r}} / 2 \pi \\
/ \mathrm{kHz}\end{array}$ & $\omega_{1} / 2 \pi$ \\
\hline$\sigma_{\mathrm{HC}}$ & 17.2 & 512 & 3 & 2(on/off) & †10s & 150 & 5 & ${ }^{*} 0.1 \mathrm{~W}$ \\
\hline $\bar{R}$ & 17.2 & 256 & 1 & 21 & $5 \mathrm{~ms} / 4 \mathrm{~s}$ & 150 & 5 & $7.8 \mathrm{kHz}$ \\
\hline$R_{1}$ & 6.1 & 256 & 3 & 21 & $5 \mathrm{~ms} / 4 \mathrm{~s}$ & 175 & 10 & $8.2 \mathrm{kHz}$ \\
\hline$R_{1}$ & 44.8 & 1024 & 4 & 21 & $5 \mathrm{~ms} / 4 \mathrm{~s}$ & 100 & 5 & $5.3 \mathrm{kHz}$ \\
\hline$R_{1 \rho}$ & 17.2 & 256 & $2(4)$ & 18 & $0.2 \mathrm{~ms} / 100 \mathrm{~ms}$ & 150 & 5 & $22.1 \mathrm{kHz}$ \\
\hline$R_{1 \rho}$ & 17.2 & 256 & $2(2)$ & 18 & $0.2 \mathrm{~ms} / 100 \mathrm{~ms}$ & 150 & 5 & $12.0 \mathrm{kHz}$ \\
\hline$R_{1 \rho}$ & 17.2 & 256 & $2(2)$ & 18 & $0.2 \mathrm{~ms} / 100 \mathrm{~ms}$ & 150 & 5 & $7.0 \mathrm{kHz}$ \\
\hline DIPSHIFT & 17.2 & 512 & 5 & 33 & - & 150 & 2 & $65 \mathrm{kHz}$ \\
\hline
\end{tabular}

${ }^{*}$ value not calibrated. ${ }^{1} \mathrm{H}$ saturation established experimentally

${ }^{\dagger}$ value is the length of the steady-state NOE delay (at least $3.4 x{ }^{13} \mathrm{C} \mathrm{T}_{1}$ )

${ }^{\ddagger}$ value in parenthesis is the number of separate experiments performed with different offsets

Relaxation rate constants are extracted from the series of 1D data by first fitting a reference spectrum using INFOS, ${ }^{16}$ and then fixing the positions and widths of the fitted peaks, but allowing amplitude and relaxation rate constants to vary (INFOS FitTrace function). All series were fitted to exponentially decaying functions ( $\exp \left(-t^{*} R\right)$ or $1-\exp \left(-t^{*} R\right)$, the latter for $T_{1}$ recovery). For DIPSHIFT, fitting was used to extract amplitudes from each 1D spectra, which were then separately fit in MATLAB, to explicit simulations of the DIPSHIFT sequence (simulation script provided as DIPSHIFT_sim.m in the MATLAB folder on https://github.com/alsinmr/POPC_frames_archive). 


\section{Data analysis}

\section{Detector analysis}

Both experimental data and MD-derived correlation functions are analysed using the detector approach, described previously. ${ }^{17}$ That is, experimental data is fitted with detector responses, minimizing

$$
\min \sum_{\zeta} \sum_{n} \frac{\left(R_{\zeta}^{\text {exp. }}-[\mathbf{r}]_{\zeta, n} \rho_{n}^{(\theta, S)}\right)^{2}}{\sigma\left(R_{\zeta}\right)^{2}}
$$

where $\mathbf{r}$ is a matrix that has been optimized so that we obtain the set of detector sensitivities given in Fig. 1A. The $\mathbf{r}$ matrices used for experimental analysis can be found in SI Tables 1-4. Resulting data fits found in SI Table 5.

We also use detectors to characterize dynamics from MD-derived correlation functions. This differs from the previous implementation, where correlation functions were first analysed with an inverse Laplace transform, and main text eq. (1) was computed explicitly. ${ }^{18}$ The requirement for application of detector analysis is a linear relationship between the distribution of motion, $\left(1-S^{2}\right) \theta(z)$, and the measured parameter, for an experimental relaxation rate constant, and a time point in a correlation function, these are

$$
\begin{aligned}
& R_{\zeta}^{(\theta, S)}=\left(1-S^{2}\right) \int_{-\infty}^{\infty} \theta(z) R_{\zeta}(z) d z \\
& C(t)=S^{2}+\left(1-S^{2}\right) \int_{-\infty}^{\infty} \theta(z) \underbrace{\exp \left(-t /\left(10^{z} \cdot 1 s\right)\right)}_{R_{C(t)}(z)} d z
\end{aligned}
$$

We compare the relationship of an experimental relaxation rate constant to $\left(1-S^{2}\right) \theta(z)$, to the relationship of a time point of the correlation function to $\left(1-S^{2}\right) \theta(z)$. The two are essentially the same form, excepting the offset term, $S^{2}$. When fitting correlation functions, the term $S^{2}$ can be neglected: for a finite trajectory, it is not possible to differentiate the nondecaying fraction of the correlation function $\left(S^{2}\right)$ from the very slowly decaying components. If we allow for correlation times at significantly longer than the trajectory, contributions from $S^{2}$ are simply absorbed into these long correlation times. Then, the sensitivity of a time point extracted from the trajectory is $R_{c(t)}(z)=\exp \left(-t /\left(10^{z} \cdot 1 \mathrm{~s}\right)\right)$. These sensitivities may be used to optimize detectors, as previously described for experimental sensitivities (see SI of ref. 16). Then we optimize the MD sensitivities to match the first four experimentally-derived detectors. Also see SI section 1.4 for further discussion. 


\section{Frames analysis}

The total correlation function may be calculated as

$$
\begin{aligned}
& C(t)=\left\langle D_{00}^{2}\left(\Omega_{\tau++\tau}^{v}\right)\right\rangle_{\tau} \\
& C\left(t_{n}\right)=\frac{1}{N-n} \sum_{m=0}^{N-n-1} \frac{3\left(\mathbf{v}\left(\tau_{m}\right) \cdot \mathbf{v}\left(\tau_{m+n}\right)\right)^{2}-1}{2}
\end{aligned}
$$

$D_{00}^{2}\left(\Omega_{\tau, t+\tau}^{\mathrm{v}}\right)$ is the $(0,0)$ component of the rank-2 Wigner rotation matrix element, operating on the Euler angles which rotate from the direction of an interaction tensor (collinear with the $\mathrm{H}-\mathrm{C}$ or $\mathrm{C}=\mathrm{O}$ bond) at some time $\tau$ to some later time $t+\tau$ (Euler angles given in the frame of the bond at the initial time, $\tau) . D_{00}^{2}\left(\Omega_{\tau, t+\tau}^{v}\right)$ is averaged over all pairs of time points separated by $t$, indicated by the brackets, $\langle\ldots\rangle_{\tau}$, with averaging over the initial time, $\tau$. The latter equation gives a practical implementation of this formula, where $\mathbf{v}(\tau)$ are normalized vectors pointing in the direction of the bond $\left(D_{00}^{2}(\Omega)\right.$ is equal to $\left(3 \cos ^{2} \beta-1\right) / 2$, where the dot product of the normalized vectors yields $\cos \beta$ ). Eq. (M3) is used for calculating the correlation functions used for comparison to experimental analysis in Fig. 1.

In order to separate motions, we assume that we can define some frame for which a bond reorients due to reorientation of the frame. Then, the motion of the bond is the product of rotations within the frame, that is, motion that is not correlated with the frame motion, and rotations of the frame. Suppose we have a bond at time $\tau$, expressed in its own frame (that is, $\left.\mathbf{v}^{\mathbf{v}}(\tau)=[0,0,1]\right)$, and the same bond at some later time, given in the same frame, $\mathbf{v}^{\mathbf{v}}(t+\tau)$ (we use the superscript, $\mathbf{v}$, to indicate the frame of the bond at the initial time). Then, the total rotation is given by

$$
\begin{aligned}
& \mathbf{v}^{\mathbf{v}}(t+\tau)=\mathbf{R}_{Z Y Z}\left(\Omega_{\tau, t+\tau}^{\mathrm{v}}\right) \cdot \underbrace{\mathbf{v}^{\mathrm{v}}(\tau)}_{=[0,0,1]} . \\
& =\mathbf{R}_{Z Y Z}\left(\Omega_{\tau, t+\tau}^{\mathrm{v} f}\right) \cdot \mathbf{R}_{Z Y Z}\left(\Omega_{\tau, t+\tau}^{\mathbf{v}-f}\right) \cdot \mathbf{v}^{\mathbf{v}}(\tau)
\end{aligned} .
$$

We have simply broken the total rotation between the two vectors into two components: the first $\left(\Omega_{\tau f+\tau}^{v-f}\right)$ is the rotation of the bond due to motion within the frame $(-f$ indicates frame motion is removed), and the second $\left(\Omega_{\tau \neq+\tau}^{v f}\right)$ is the rotation of the bond due to motion of the frame (indicated by :f). Then, the same rotation occurring in (M3) may be separated the same way, using the usual rules of spherical tensor rotations, as

$$
\begin{aligned}
& C(t)=\left\langle D_{00}^{2}\left(\Omega_{\tau f+\tau}^{\mathrm{v}}\right)\right\rangle_{\tau} \\
& C(t)=\sum_{p=-2}^{2}\left\langle D_{\rho 0}^{2}\left(\Omega_{\tau \neq+\tau}^{\mathrm{v} f}\right) D_{0 \rho}^{2}\left(\Omega_{\tau \neq+\tau}^{\mathrm{v}-f}\right)\right\rangle_{\tau}
\end{aligned}
$$


At this stage, we first assume statistical independence of motion within the frame and motion of the frame, ${ }^{20,21}$ such that $\left\langle D_{p 0}^{2}\left(\Omega_{\tau, t+\tau}^{\mathbf{v} f}\right) D_{0 p}^{2}\left(\Omega_{\tau, t+\tau}^{\mathbf{v} f}\right)\right\rangle_{\tau}=\left\langle D_{p 0}^{2}\left(\Omega_{\tau, t+\tau}^{\mathbf{v} f}\right)\right\rangle_{\tau}\left\langle D_{0 p}^{2}\left(\Omega_{\tau, t+\tau}^{\mathbf{v}-f}\right)\right\rangle_{\tau}$.

$$
C(t)=\sum_{\rho=-2}^{2}\left\langle D_{\rho 0}^{2}\left(\Omega_{\tau f+\tau}^{v f}\right)\right\rangle_{\tau}\left\langle D_{0 \rho}^{2}\left(\Omega_{\tau, t+\tau}^{v-f}\right)\right\rangle_{\tau}
$$

Second, we assume timescale separation, specifically, we require that there is some time, $t_{1}$, such that for $t<t_{1},\left\langle D_{p 0}^{2}\left(\Omega_{\tau, t+\tau}^{v f f}\right)\right\rangle_{\tau} \approx \delta_{p}$, that is, the orientation of the frame has not evolved significantly. For $t>t_{1}$, we require that the shape, although not necessarily the magnitude of the residual tensor due to motion within the frame stops evolving, such that $\left\langle D_{0 p}^{2}\left(\Omega_{\tau f+\tau}^{\mathbf{v} f}\right\rangle_{t} I\left\langle D_{00}^{2}\left(\Omega_{\tau, t+\tau}^{\mathbf{v}-f}\right\rangle_{\tau} \approx \lim _{t \rightarrow \infty}\left[\left\langle D_{0 p}^{2}\left(\Omega_{\tau f+\tau}^{\mathbf{v} f}\right\rangle_{t} I\left\langle D_{00}^{2}\left(\Omega_{\tau f+\tau}^{\mathbf{v}-f}\right\rangle_{\tau}\right]\right.\right.\right.\right.$. The correlation function for these two limits becomes

$$
\begin{aligned}
& t<t_{1} \\
& C(t)=\sum_{p=-2}^{2} \delta_{p}\left\langle D_{0 \rho}^{2}\left(\Omega_{\tau, t+\tau}^{\mathbf{v}-f}\right)\right\rangle_{\tau}=\left\langle D_{\infty}^{2}\left(\Omega_{\tau, t+\tau}^{\mathbf{v}-f}\right)\right\rangle_{\tau} \\
& t>t_{1} \\
& C(t)=\left\langle D_{\infty}^{2}\left(\Omega_{\tau, t+\tau}^{\mathbf{v}-f}\right)\right\rangle_{\tau} \sum_{p=-2}^{2}\left\langle D_{p 0}^{2}\left(\Omega_{\tau, t+\tau}^{\mathbf{v} f}\right)\right\rangle_{\tau} \lim _{t \rightarrow \infty} \frac{\left\langle D_{0 \rho}^{2}\left(\Omega_{\tau, t+\tau}^{\mathbf{v}-f}\right)\right\rangle_{\tau}}{\left\langle D_{\infty}^{2}\left(\Omega_{\tau, t+\tau}^{\mathbf{v}-f}\right)\right\rangle_{\tau}}
\end{aligned}
$$

We may then define two correlation functions, $C^{\mathbf{v} f}(t)$, which describes motion within the frame, and $C^{\mathbf{v} f}(t)$, which describes motion of the frame, whose product yields $C(t)$ at all times, $t$.

$$
\begin{aligned}
& C^{\mathbf{v}-f}(t)=\left\langle D_{\infty}^{2}\left(\Omega_{\tau, t+\tau}^{\mathbf{v}-f}\right)\right\rangle_{\tau} \\
& C^{\mathbf{v} f}(t)=\sum_{p=-2}^{2}\left\langle D_{\rho 0}^{2}\left(\Omega_{\tau, t+\tau}^{\mathbf{v} f}\right)\right\rangle_{\tau} \lim _{t \rightarrow \infty} \frac{\left\langle D_{0 \rho}^{2}\left(\Omega_{\tau, t+\tau}^{\mathbf{v}-f}\right)\right\rangle_{\tau}}{\left\langle D_{\infty}^{2}\left(\Omega_{\tau, t+\tau}^{\mathbf{v} f}\right)\right\rangle_{\tau}} \\
& C(t)=C^{\mathbf{v} f f}(t) \cdot C^{\mathbf{v}-f}(t)
\end{aligned}
$$

In this formulation, the terms $\lim _{t \rightarrow \infty}\left\langle D_{0 \rho}^{2}\left(\Omega_{\tau \neq+\tau}^{2}\right)\right\rangle_{\tau}$ describe residual tensors resulting from all motion within the frame, similar to what is shown in Fig. 4. Normalization with $\lim _{t \rightarrow \infty}\left\langle D_{\infty}^{2}\left(\Omega_{\tau \neq+\tau}^{2}\right)\right\rangle_{\tau}$ shows us that it is only reshaping/reorientation of this tensor that must be timescale separated from reorientation of the frame itself, whereas the absolute magnitude may vary. That is, $\delta$, the anisotropy may continue to decay, but variation of $\eta$, the asymmetry, and the Euler angles must remain timescale separated from frame reorientation. 
Practically, $C^{\mathbf{v} f}(t)$ is obtained by evaluating (M3) in a time-dependent frame, which is aligned such that motion of the frame is removed. Take $\mathbf{v}_{\mathrm{Z}}(\tau)$ to be a normalized vector giving the direction of the bond, and terms $v_{\alpha}^{f}(\tau)$ to be axes of the frame (we use $v_{\alpha}^{f}(\tau)$ as shorthand for each of the three vectors $\left.v_{\mathrm{X}}^{f}(\tau), v_{\mathrm{Y}}^{f}(\tau), v_{\mathrm{Z}}^{f}(\tau)\right)$. We take $\Omega_{\tau}^{f}$ to be the set of Euler angles such that we obtain the $v_{\alpha}^{f}(\tau)$ by applying $\Omega_{\tau}^{f}$ to $x, y$, or $z$.

$$
\begin{aligned}
& \mathbf{R}_{Z Y Z}\left(\Omega_{\tau}^{f}\right) \cdot[1,0,0]^{\prime}=v_{X}^{f}(\tau) \\
& \mathbf{R}_{Z Y Z}\left(\Omega_{\tau}^{f}\right) \cdot[0,1,0]^{\prime}=v_{Y}^{f}(\tau) . \\
& \mathbf{R}_{Z Y Z}\left(\Omega_{\tau}^{f}\right) \cdot[0,0,1]^{\prime}=v_{Z}^{f}(\tau)
\end{aligned}
$$

Then, we apply $\mathbf{R}_{\mathrm{ZYZ}}^{-1}\left(\Omega_{\tau}^{f}\right)$ to $\mathbf{v}_{\mathrm{Z}}(\tau)$, and subsequently calculate $\left\langle D_{\infty}^{2}\left(\Omega_{\tau, t+\tau}^{\mathbf{v}-f}\right)\right\rangle_{\tau}$ :

$$
\begin{aligned}
& \mathbf{R}_{Z Y Z}^{-1}\left(\Omega_{\tau}^{f}\right) \cdot \mathbf{v}_{Z}(\tau)=\mathbf{v}_{Z}^{-f}(\tau) \\
& C^{\mathbf{v}-f}\left(t_{n}\right)=-\frac{1}{2}+\frac{1}{N-n} \frac{3}{2} \sum_{m=0}^{N-n-1}\left(\mathbf{v}_{Z}^{f}\left(\tau_{m}\right) \cdot \mathbf{v}_{Z}^{-f}\left(\tau_{m+n}\right)\right)^{2}
\end{aligned}
$$

Calculation of $C^{v f}(t)$ is considerably more complex, requiring several terms depending on various elements of the Wigner rotation matrix elements. First, we require a consistent definition of the frame of the bond, given by time-dependent axes $\mathbf{v}_{\alpha}(\tau)$. As stated before, $\mathbf{v}_{\mathrm{Z}}(\tau)$ should lie along the bond. Then, for an $\mathrm{H}-\mathrm{C}$ bond, we take a $\mathrm{C}-\mathrm{C}$ bond (containing the $\mathrm{C}$ from the $\mathrm{H}-\mathrm{C}$ bond) to lie in the $x z$-plane of the bond frame. From this, we can calculate $\mathbf{v}_{\mathbf{X}}(\tau)$ and $\mathbf{v}_{\mathbf{Y}}(\tau)$ (their definitions are arbitrary, as long as those definitions remain consistent, and the axes are orthonormal). To obtain $C^{\mathrm{vf}}(t)$, we require the terms $\left\langle D_{0 p}^{2}\left(\Omega_{\tau \neq+\tau}^{v-f}\right)\right\rangle_{\tau}$, describing the residual tensor of motion in the frame. These can be obtained by first finding the Euler angles $\Omega_{\tau}^{\mathbf{v}-\boldsymbol{f}}$ such that

$$
\begin{aligned}
& \mathbf{R}_{Z Z Z}\left(\Omega_{\tau}^{\mathbf{v}-f}\right) \cdot[1,0,0]^{\prime}=\mathbf{v}_{\mathrm{X}}^{-f}(\tau) \\
& \mathbf{R}_{Z \mathrm{ZZ}}\left(\Omega_{\tau}^{\mathbf{v}-f}\right) \cdot[0,1,0]^{\prime}=\mathbf{v}_{\mathrm{Y}}^{-f}(\tau) . \\
& \mathbf{R}_{Z \mathrm{ZZ}}\left(\Omega_{\tau}^{\mathbf{v}-f}\right) \cdot[0,0,1]^{\prime}=\mathbf{v}_{\mathrm{Z}}^{-f}(\tau)
\end{aligned}
$$

Using the resulting Euler angles, we then apply $\mathbf{R}_{Z Y Z}^{-1}\left(\Omega_{\tau}^{\mathbf{v}-f}\right)$ to the vectors $\mathbf{v}_{\alpha}^{-f}(t+\tau)$; the result is denoted as $\mathbf{v}_{\alpha}^{\mathbf{v}-f}(t+\tau)$. These axes define the frame of the bond at time $t+\tau$, represented in the frame of the bond at time $\tau$, where the motion of frame $f$ is removed. Then, we finally define $\Omega_{\tau \neq+\tau}^{v-f}$ to be the set of Euler angles that rotate to the bond from its frame at time $\tau$ to its orientation (in that frame), at time $t+\tau$. These may be inserted into 
the terms $\left\langle D_{0 \rho}^{2}\left(\Omega_{\tau, t+\tau}^{\mathbf{v}-f}\right)\right\rangle_{\tau}$ required in (M8). To obtain the limit as $t \rightarrow \infty$, we assume the MD orientational sampling is representative of the thermal equilibrium, and therefore pair all time points with all other time points, and take the average with equal weighting.

The rotation of the bond within the frame is calculated in the frame of the bond at its initial time. Then, rotation of the bond due to motion of the frame should be calculated in that same frame. To achieve this, we need the Euler angles rotating the bond from its initial orientation at time $\tau$, defined by $\mathbf{v}_{\alpha}(\tau)$, to its orientation at time $t+\tau$, but where the new orientation is only the result of motion of the frame (motion in the frame removed). This is obtained by taking the $\mathbf{v}_{z}^{-f}(\tau)$, where motion of frame $f$ is removed at time $\tau$. Multiplication of each term by $\mathbf{R}_{Z Y Z}\left(\Omega_{t+\tau}^{f}\right)$ then yields the $\mathbf{v}_{\alpha}^{f}(t+\tau)$, which have been reoriented from time $\tau$ only by motion of the frame. This can be seen below, where we see that the resulting terms are the result of the change in orientation due to the frame motion between times $\tau$ and $t+\tau$.

$$
\mathbf{v}_{\alpha}^{f}(t+\tau)=\mathbf{R}_{\mathrm{ZYZ}}\left(\Omega_{t+\tau}^{f}\right) \cdot \mathbf{v}_{\alpha}^{-f}(\tau)=\mathbf{R}_{\mathrm{ZYZ}}\left(\Omega_{i+\tau}^{f}\right) \cdot \underbrace{\mathbf{R}_{\mathrm{ZYZ}}^{-1}\left(\Omega_{\tau}^{f}\right) \cdot \mathbf{v}_{\alpha}(\tau)}_{\mathbf{v}_{\alpha}^{-f}(\tau)} .
$$

As before, we find the Euler angles defining the frame of the bond at time $\tau, \Omega_{\tau}^{\mathbf{v}}$, and apply $\mathbf{R}_{Z Y Z}^{-1}\left(\Omega_{\tau}^{\mathbf{v}}\right)$ to the $\mathbf{v}_{\alpha}^{f}(t+\tau)$, yielding $\mathbf{v}_{\alpha}^{\mathbf{v} f f}(t+\tau)$, which are the vectors at time $t+\tau$, in the frame of the bond at time $\tau$, where rotation between these times is due only to motion of the frame. Then, we finally must obtain the Euler angles yielding the $\mathbf{v}_{\alpha}^{\mathbf{v} f}(t+\tau)$, defined as $\Omega_{\tau f+\tau}^{v f}$, and insert these into the terms $\left\langle D_{p 0}^{2}\left(\Omega_{\tau, t+\tau}^{v f}\right)\right\rangle_{\tau}$. The resulting terms may be used in eq. (M8), to obtain the correlation function due to motion of the frame, $C^{\mathbf{v f}}(t)$.

This procedure may also be applied iteratively. Suppose we have two frames, denoted $f$ and $F$. Then, motion within frame $f$ is obtained as before, yielding $C^{\mathbf{v}-f}(t)$. Motion due to frame $f$ is obtained by removing motion of frame $F$ first from both the bond vectors, $\mathbf{v}_{\alpha}^{-F}(\tau)$, and the vectors defining the axis of the frame $v_{\alpha}^{f-F}(\tau)$, and applying the procedure as described in the preceding paragraphs, yielding $C^{\mathbf{v} f-F}(t)$. Finally, motion due to frame $F$ is obtained by finding residual tensors within frame $F$ (we no longer need frame $f$ at this stage), and again applying the above procedure using the frame F. For each frame used, we require statistical independence of motion of the frame and motion in the frame, and require timescale separation of residual tensor reorientation/reshaping due to motion in the frame and motion of the frame. Note that residual tensors in Fig. 4 are obtained for 
intermediate frames using the procedure described for obtaining the components $\left\langle D_{\rho 0}^{2}\left(\Omega_{\tau, t+\tau}^{\mathrm{vf}}\right)\right\rangle_{\tau}$, except that we use the terms $D_{0 p}^{2}(\Omega)$ of the Wigner rotation matrix.

\section{Constructing a dynamic landscape}

Detector responses are defined by main text eq. (1), and so for a given $\left(1-S^{2}\right) \theta(z)$, one may numerically integrate $(1)$ to calculate $\rho_{n}^{(\theta, S)}$. Distributions may be fitted to the MDderived values shown in SI Fig. 12, which we achieved by performing a grid search over correlation time and distribution width, while optimizing the amplitude at every grid element, yielding a correlation function given by a discrete distribution:

$$
C^{1}(t)=S_{1}^{2}+\left(1-S_{1}^{2}\right) \sum_{i} A_{1, i} \exp \left(-t / \tau_{i}\right)
$$

Then, the product of two frames is

$$
\begin{aligned}
& C^{1}(t)=\left[S_{1}^{2}+\left(1-S_{1}^{2}\right) \sum_{i} A_{1, i} \exp \left(-t / \tau_{i}\right)\right] \cdot\left[S_{2}^{2}+\left(1-S_{2}^{2}\right) \sum_{i} A_{1, i} \exp \left(-t / \tau_{i}\right)\right] \\
& =S_{1}^{2} S_{2}^{2}+\sum_{i}\left[S_{1}^{2} A_{2, i} \exp \left(-t / \tau_{i}\right)+S_{2}^{2} A_{1, i} \exp \left(-t / \tau_{i}\right)\right]+\sum_{i} \sum_{j} A_{1, i} A_{2, j} \exp \left(-t \frac{\tau_{i} \tau_{j}}{\tau_{i}+\tau_{j}}\right) . \\
& =S_{12}^{2}+\left(1-S_{12}^{2}\right) \sum_{i} A_{12, i} \exp \left(-t / \tau_{i}\right) \exp \left(-t / \tau_{i}\right)
\end{aligned}
$$

The resulting amplitudes and correlation times can be numerically re-binned to obtain the new distribution, and the process is repeated to obtain the product of all motions as shown in SI Fig. 16. Finally, we use NMR detector responses to refine the MD-derived result, by scaling the internal correlation time (or times) by a constant factor for all positions corresponding to each resolved resonance in the NMR spectrum, with results in Fig. 5.

\section{References}

1. Jorgensen, W. L., Chandrasekhar, J., Madura, J. D., Impey, R. W. \& Klein, M. L. Comparison of simple potential functions for simulating liquid water. J. Chem. Phys. vol. 79 926-935 (1983).

2. Jo, S., Kim, T., Iyer, V. G. \& Im, W. CHARMM-GUI: A web-based graphical user interface for CHARMM. Journal of Computational Chemistry 29, 1859-1865 (2008).

3. Wu, E. L. et al. CHARMM-GUI Membrane Builder toward realistic biological membrane simulations. Journal of Computational Chemistry 35, 1997-2004 (2014).

4. Jo, S., Lim, J. B., Klauda, J. B. \& Im, W. CHARMM-GUI Membrane Builder for Mixed Bilayers and Its Application to Yeast Membranes. Biophysical Journal 97, 50-58 (2009).

5. Brooks, B. R. et al. CHARMM: The biomolecular simulation program. Journal of Computational Chemistry 30, 1545-1614 (2009).

6. Lee, J. et al. CHARMM-GUI Input Generator for NAMD, Gromacs, Amber, Openmm, and CHARMM/OpenMM Simulations using the CHARMM36 Additive Force Field. Biophysical Journal 110, 641a (2016).

7. Klauda, J. B. et al. Update of the CHARMM All-Atom Additive Force Field for Lipids: Validation on Six Lipid Types. J. Phys. Chem. B 114, 7830-7843 (2010). 
8. Hess, B., Bekker, H., Berendsen, H. J. C. \& Fraaije, J. G. E. M. LINCS: A Linear Constraint Solver for Molecular Simulations. J. Comput. Chem. vol. 18 1463-1472 (1997).

9. Tiemann, J. K. S., Guixà-González, R., Hildebrand, P. W. \& Rose, A. S. MDsrv: viewing and sharing molecular dynamics simulations on the web. Nat Methods 14, 1123-1124 (2017).

10. Fung, B. M., Khitrin, A. K. \& Ermolaev, K. An improved broadband decoupling sequence for liquid crystals and solids. J Magn Reson 142, 97-101 (2000).

11. Krushelnitsky, A., Gauto, D., Rodriguez Camargo, D. C., Schanda, P. \& Saalwachter, K. Microsecond motions probed by near-rotary-resonance R1rho(15)N MAS NMR experiments: the model case of protein overall-rocking in crystals. J Biomol NMR vol. 71 53-67 (2018).

12. Bielecki, A., Kolbert, A. C., De groot, H. J. M., Griffin, R. G. \& Levitt, M. H. FrequencySwitched Lee-Goldburg Sequences in Solids. in Advances in Magnetic and Optical Resonance (ed. Warren, W. S.) vol. 14 111-124 (Academic Press, 1990).

13. Smith, A. A. INFOS: spectrum fitting software for NMR analysis. J Biomol NMR 67, 7794 (2017).

14.Smith, A. A., Ernst, M. \& Meier, B. H. Optimized 'detectors' for dynamics analysis in solid-state NMR. J. Chem. Phys. vol. 148045104 (2018).

15. Smith, A. A., Ernst, M., Riniker, S. \& Meier, B. H. Localized and collective motions in HET-s(218-289) fibrils from combined NMR relaxation and MD simulation. Angew. Chem. Int. Ed. vol. 58 9483-9488 (2019).

16. Smith, A. A., Ernst, M., Meier, B. H. \& Ferrage, F. Reducing bias in the analysis of solution-state NMR data with dynamics detectors. J Chem Phys vol. 151034102 (2019).

17. Lipari, G. \& Szabo, A. Model-free approach to the interpretation of nuclear magnetic resonance relaxation in macromolecules. 1. Theory and range of validity. J. Am. Chem. Soc. vol. 104 4546-4559 (1982).

18. Halle, B. \& Wennerström, H. Interpretation of magnetic resonance data from water nuclei in heterogeneous systems. J. Chem. Phys. vol. 75 1928-1943 (1981). 


\section{Supplementary Files}

This is a list of supplementary files associated with this preprint. Click to download.

- tensorperpMOIHGBBintern.mov

- detperpMOIHGBBintern.mov

- detlib.mov

- tensorparaMOI.mov

- dettotal.mov

- detall.mov

- tensorMOIHGBB.mov

- tensortotal.mov

- DynamicLSSI.pdf

- detparaMOI.mov

- detMOIHGBB.mov

- tensorlib.mov

- flatsmithnrsoftwarepolicy.pdf

- smithreportingsummarycomplete.pdf 